\title{
Tangential connection
}

\author{
Massimiliano Mella \\ (Communicated by A. Sommese)
}

\begin{abstract}
Let $X \subset \mathbb{P}^{N}$ be a smooth variety. The embedding in $\mathbb{P}^{n}$ gives naturally rise to the notion of embedded tangent spaces. That is the locus spanned by tangent lines to a point $x \in X$. Generally the embedded tangent space intersects the variety $X$ only at the point $x$. In this paper I am interested in those $X$ for which this intersection, for $x \in X$ general, is a positive dimensional subvariety. The results of this paper support the conjecture that these varieties are built out of some special varieties.
\end{abstract}

\section{Introduction}

Let $X \subset \mathbb{P}^{N}$ be a smooth variety. The embedding in $\mathbb{P}^{n}$ gives naturally rise to the notion of embedded tangent spaces. That is the locus spanned by tangent lines to a point $x \in X$. Generally the embedded tangent space intersects the variety $X$ only at the point $x$. In this paper I am interested in those $X$ for which this intersection, for $x \in X$ general, is a positive dimensional subvariety. The results of this paper support the conjecture that these varieties are built out of some special varieties that I call tangentially connected, see Definition 2.3. Actually I prove this under mild restrictions. The main technique used is the Campana, Kollár-Miyaoka-Mori theory of algebraic relations, [7]. This provides the varieties studied with a proper rational map onto a lower dimensional variety. The main difficulty is that, unlike the usual algebraic relations, it is not immediate that the general fibres of this map enjoy any relevant tangential property. The first main result is that, under some conditions, see Theorem 2.6, fibres of this map are tangentially connected. Therefore the study of varieties with positive dimensional tangential restriction is reduced to that of tangentially connected varieties. With the help of a slicing method, Theorem 2.14, I then start a classification of $T C$ varieties. In doing this a quite surprising characterization of quadric 3-folds is obtained, and a classical result of del Pezzo, [2], is reinterpreted. Then some properties of TC 3-folds are proved and a conjectural classification is proposed. In the Appendix, via adjunction theory, I classify varieties with respect to the degree of curves contained in the image of the Gauss map. 


\section{Notations and preliminaries}

Let $X \subset \mathbb{P}^{N}$ be a smooth projective variety over the complex field. Fix a point $x \in X$, then $\mathbb{T}_{x} X$ denotes the projective tangent space to $X$ at $x$. That is the linear space spanned by tangent lines to $X$ at $x$. Let $Z \subset \mathbb{P}^{N}$ any subscheme; then $\langle Z\rangle$ stands for the linear span of $Z$. That is the smallest linear space contained in $\mathbb{P}^{N}$ containing $Z$.

Definition 1.1. Let $x \in X$ be a general point and assume that $X \cap \mathbb{T}_{x} X$ has positive dimension. Then $X$ is said to have a positive dimensional tangential restriction.

Assume that $X$ has a positive dimensional tangential restriction. Let $W_{x}$ be an irreducible component of $\left(X \cap \mathbb{T}_{x} X\right)_{\text {red. }}$. These data single out a special family of subschemes of $X$, in the following way. Let $P\left(W_{x}\right)$ be the Hilbert polynomial of $W_{x} \subset X \subset \mathbb{P}^{N}$. Since $x \in X$ is general, there is a dense subset $A \subset X$, the complement of countably many closed subsets, such that for any $y \in A$ the subscheme $\left(\mathbb{T}_{y} X \cap X\right)_{\text {red }}$ has an irreducible component $W_{y}$ with Hilbert polynomial $P\left(W_{x}\right)$. Let $P=P\left(W_{x}\right)$ and

$$
V^{0}=\left\{\left[W_{y}\right] \mid y \in A\right\} \subset \operatorname{Hilb}_{P}(X) .
$$

Finally consider

$$
V \subseteq \overline{V^{0}} \subset \operatorname{Hilb}_{P}(X),
$$

an irreducible component of maximal dimension of the closure.

By general theory of the Hilbert scheme, see for instance [7], there exists a universal family $U$ over $V$ with proper morphisms

$$
V \stackrel{q}{\longleftarrow} U \stackrel{p}{\rightarrow} X .
$$

In our hypothesis $p$ is surjective and $q$ is connected. That is $V$ is a dominant family of connected cycles.

Definition 1.2. In this notation for any $Z \subset X$ let $\mathbb{S}_{Z}=p\left(q^{-1}\left(\left(q\left(p^{-1}(Z)\right)\right)\right)\right)$. That is the locus of cycles parametrized by $V$ and passing through $Z$.

Definition 1.3. In the following a scroll is always understood as a classical (geometric) scroll over a variety $B$. That is the birational image of a projective bundle $\mathbb{P}:=\mathbb{P}_{B}(\mathcal{E})$ via the spanned linear system $\left|\mathcal{O}_{\mathbb{P}}(1)\right|$.

There is a very handy criterion to recognize scrolls over curves.

Theorem 1.4 ([8]). Let $X \subset \mathbb{P}^{N}$ be an irreducible variety of dimension $k$. Let $\Sigma \subset$ $\mathbb{G}(1, N)$ be a component of maximal dimension of the variety of lines contained in $X$. Then

i) if $\operatorname{dim} \Sigma=2 k-2$, then $X$ is a linearly embedded $\mathbb{P}^{k}$,

ii) if $\operatorname{dim} \Sigma=2 k-3$, then $X$ is either a quadric or a scroll over a curve.

The following is very useful, see [6] and [3, Theorems 2.1 and 3.2]. 
Theorem 1.5. Let $X \subset \mathbb{P}^{N}$ be a smooth n-fold. Assume that the defect of $X$ is $k$ (i.e. $\operatorname{dim} X^{*}=N-1-k$ ). Let $q \in X$ be a general point and $H$ a general tangent hyperplane at $q$. Then $H_{\mid X}$ is singular along a k-dimensional linear space $L \ni q$ and the tangent cone at $p \in L$ is a quadric of rank $n-k$.

\section{Tangential connectedness}

Let $X \subset \mathbb{P}^{N}$ be a smooth variety with positive dimensional tangential restriction of dimension $n$. Fix a general point $x \in X$, and an irreducible component $W_{x} \subset\left(\mathbb{T}_{x} X \cap\right.$ $X)_{\text {red }}$. As in Section 1 this gives rise to the following data. A closed subscheme $V \subset$ $\operatorname{Hilb}(X)$ and an incidence correspondence with proper morphisms

$$
V \stackrel{q}{\leftarrow} U \stackrel{p}{\rightarrow} X,
$$

where $U$ is the universal family and $p$ is the natural projection.

The aim is to use

$$
V \stackrel{q}{\longleftarrow} U \stackrel{p}{\rightarrow} X
$$

to produce an algebraic equivalence relation following Campana and Kollár-MiyaokaMori. To do this I use the technique developed in [7]. Taking normal forms, [7, IV (4.4.5)], I obtain a proper and connected prerelation

$$
\left(V^{\prime} \stackrel{s}{\leftarrow} U^{\prime} \stackrel{w}{\rightarrow} X, V^{\prime} \stackrel{\sigma}{\rightarrow} U^{\prime}\right) .
$$

Definition 2.1. The prerelation

$$
\left(V^{\prime} \stackrel{s}{\leftarrow} U^{\prime} \stackrel{w}{\rightarrow} X, V^{\prime} \stackrel{\sigma}{\rightarrow} U^{\prime}\right)
$$

gives rise to an algebraic relation, [7, IV (4.8)]. I call this relation $T_{W} R(X)$.

Two points on $X$ are related by $T_{W} R(X)$ if there exists a finite connected chain of cycles $\left[W_{i}\right] \in V$ that joins them. Furthermore there exists an open subvariety $X^{0} \subset X$ and a proper morphism $\pi^{0}: X^{0} \rightarrow Z^{0}$ with connected fibres of positive dimension such that [7, IV (4.16)]:

- $T_{W} R(X)$ restricts to an equivalence relation on $X^{0}$,

- $\pi^{-1}(z)$ coincides with a $T_{W} R(X)$ equivalence class for every $z \in Z^{0}$.

Definition 2.2. Any rational map $\pi: X \rightarrow Z$ such that $\pi_{\mid X^{0}}=\pi^{0}: X^{0} \rightarrow Z^{0}$ is called a $T_{W} R(X)$-map.

Definition 2.3. A smooth variety $X \subset \mathbb{P}^{N}$ is said to be tangentially connected (TC) if, given two general points $x$ and $y$ in $X$, there exists a finite set of points $z_{1}, \ldots, z_{k}$ and a connected curve $Z$ such that $Z \ni\{x\} \cup\{y\}$ and $Z \subset \bigcup_{i}\left(\mathbb{T}_{z_{i}} X \cap X\right)$. By means of the algebraic relation built in Definition 2.1 we strengthen the notion of $T C$ variety as follows. A smooth variety $X \subset \mathbb{P}^{N}$ is said to be tangentially connected with respect to the relation $T_{W} R(X), T_{W} C$ for short, if the $T_{W} R(X)$ map is constant. In other words there is a unique equivalence class of $T_{W} R(X)$ in $X^{0}$. 
Example 2.4. First examples of $T_{W} C$ varieties are $\mathbb{P}^{n}$, hypersurfaces and more generally varieties of codimension less than the dimension (consider $\mathbb{P}^{n}$ as a codimension 0 variety!). A different kind of $T C$ varieties is given by varieties which are generically covered by lines. Observe that $\mathbb{Q}^{2}$ is $T C$ but not $T_{W} C$, see also Example 2.12 .

My aim is to use this algebraic relation to study smooth projective varieties with positive dimensional tangential restriction.

Example 2.5. Unlike usual algebraic relations it is not true that the general fibre of $\pi$ is $T_{W} C$. Consider an embedded quadric fibration $X$ with relative Picard number one. The scheme $W$ is a line. Since the relative Picard number is one, all lines are in the same irreducible component of the Hilbert scheme and the $T_{W} R(X)$ map is the quadratic fibration. On the other hand the general fibre is $\mathbb{Q}^{2}$ which is not $T_{W} C$. Nonetheless observe that the fibre has positive dimensional tangential restriction and it is $T C$.

The main problem encountered in the above example is that passing from a variety to a subvariety we change the Hilbert scheme and possibly split some cycles formerly belonging to the same irreducible component. On the other hand an interesting point is that the fibre is $T C$.

The first step is to understand the relation between $\mathbb{T}_{x} X$ and $\mathbb{T}_{x} F$ where $F \subset X$ is a general fibre of a morphism.

Theorem 2.6. Let $\pi: X \rightarrow Z$ be any proper dominant morphism of fibre type with general fibre $F$. Let $x \in F \subset X$ be a point such that both $F$ and $X$ are smooth at $x$, and $Z$ is smooth at $\pi(x)$. Then

$$
\mathbb{T}_{x} X \cap F=\mathbb{T}_{x} F \cap F
$$

An ingredient to prove Theorem 2.6 is the following lemma.

Lemma 2.7. Let $S \subset \mathbb{P}^{N}$ be a scroll with structure map $\varphi: S \rightarrow B$. Assume that there exists a subvariety $Y \subset S$ such that $\varphi_{\mid Y}: Y \rightarrow B$ is dominant. Fix a point $y \in Y$ such that both $Y$ and $S$ are smooth at $y$ and $B$ is smooth at $\varphi(y)$. Let $G$ be the fibre of $\varphi$ through $y$, and $G_{Y}=G \cap Y$. Then

$$
\mathbb{T}_{y} Y \cap G=\mathbb{T}_{y} G_{Y} .
$$

Proof. By definition $\mathbb{T}_{y} Y \cap G \supset \mathbb{T}_{y} G_{Y}$. I have to prove the other inclusion. Let $\tilde{B} \subset Y$ be a subvariety with a finite dominant morphism onto $B$, locally étale at $y$. The existence of such $\tilde{B}$ is ensured by the hypothesis. Let $M=\left\langle\mathbb{T}_{y} \tilde{B}, G\right\rangle$.

We claim that $M=\mathbb{T}_{y} S$. The claim is equivalent to $\mathbb{T}_{y} \tilde{B} \cap G=\{y\}$. Assume by contradiction that there is a line $l \subset \mathbb{T}_{y} \tilde{B} \cap G$. With no restrictions I can assume that $y \in l$. Let $t \in T_{y} S$ the direction of $l$. By hypothesis $\varphi$ is regular at $y$. Let $d \varphi_{y}: T_{y} S \rightarrow T_{\varphi(y)} B$ be the differential of $\varphi$ at the point $y$. Since $l \subset G$ then $d \varphi_{y}(t)=0$, on the other hand $t \in T_{y} \tilde{B}$ therefore $d \varphi_{y}(t) \neq 0$.

To conclude observe that $\mathbb{T}_{y} Y \supset \mathbb{T}_{y} \tilde{B}$, therefore $\operatorname{dim} \mathbb{T}_{y} Y \cap G \leq \operatorname{dim} Y-\operatorname{dim} B=$ $\operatorname{dim} G_{Y}$. 
Proof of Theorem 2.6. One direction is clear. I have to prove that

$$
\mathbb{T}_{x} X \cap F \subset \mathbb{T}_{x} F \cap F
$$

Let $\mathcal{L}=\mathcal{O}_{\mathbb{P}^{N}}(1)_{\mid X} \subseteq\left|\mathcal{O}_{X}(1)\right|$. By definition

$$
\mathbb{T}_{x} X \cap F=\operatorname{Bsl}\left(\mathcal{L} \otimes \mathcal{I}_{x}^{2}\right) \cap F .
$$

Let $\mathcal{L}_{F}=\mathcal{L}_{\mid F}, f$ the dimension of the linear system $\mathcal{L}_{F}$. After maybe shrinking $Z$ to $Z^{0}$ and $X$ to a neighborhood $X^{0}$ of the fibre $F$ there exists an embedding as $Z^{0}$ schemes $X^{0} \subset \mathbb{P}_{Z^{0}}^{f}$. That is to say, we have the following embedding of $X^{0}$

$$
X^{0} \subset \mathbb{P}(\mathcal{F}) \subset \mathbb{P}^{M},
$$

for some vector bundle $\mathcal{F}$, of rank $f+1$, where

$$
\mathcal{O}_{\mathbb{P}^{M}}(1)_{\mid X^{0}}=\mathcal{L} \otimes \pi^{*} A,
$$

for some line bundle $A \in \operatorname{Pic}\left(Z^{0}\right)$. Let $G \cong \mathbb{P}^{f}$ the fibre of $\mathbb{P}(\mathcal{F})$ containing $F$. Lemma 2.7 yields

$$
\mathbb{T}_{x} X^{0} \cap G=\mathbb{T}_{x} F \subset \mathbb{P}^{M} .
$$

This yields

$$
\operatorname{Bsl}\left(\mathcal{L} \otimes \pi^{*} A \otimes \mathcal{I}_{x}^{2}\right) \cap F=\operatorname{Bsl}\left(\mathcal{L}_{F} \otimes \mathcal{I}_{x}^{2}\right) .
$$

The equality $\operatorname{dim}\left(\mathcal{L} \otimes \pi^{*} A\right)_{\mid F}=\operatorname{dim} \mathcal{L}_{F}$ and the equivalence $\mathcal{L} \otimes \pi^{*} A \otimes \mathcal{O}_{F} \sim \mathcal{L} \otimes \mathcal{O}_{F}$ force the equality between the two base loci

$$
\operatorname{Bsl}\left(\mathcal{L} \otimes \pi^{*} A \otimes \mathcal{I}_{x}^{2}\right) \cap F=\operatorname{Bsl}\left(\mathcal{L} \otimes \mathcal{I}_{x}^{2}\right) \cap F .
$$

Thus by Equations (1) and (2)

$$
\mathbb{T}_{x} X \cap F=\operatorname{Bsl}\left(\mathcal{L} \otimes \mathcal{I}_{x}^{2}\right) \cap F=\operatorname{Bsl}\left(\mathcal{L}_{F} \otimes \mathcal{I}_{x}^{2}\right)=\mathbb{T}_{x} F \cap F \subset \mathbb{P}^{N} .
$$

A direct consequence of Theorem 2.6 is the property I was looking for, at least for some class of varieties.

Theorem 2.8. Let $\pi^{0}: X^{0} \rightarrow Z^{0}$ be the $T_{W} R(X)$-fibration. Assume that one of the following is satisfied:

i) $x \in W_{x}$;

ii) $\operatorname{dim} X \leq 3$ and $\operatorname{dim} X-\operatorname{dim} Z=1$.

Then general fibres are TC.

Remark 2.9. I do not have any examples of varieties that do not satisfy condition i). Actually I conjecture that this is always the case.

Let me start with the following lemma. 
Lemma 2.10. Let $x \in X \subset \mathbb{P}^{N}$ be a general point in a smooth $k$-fold $X$. Let $C \ni x$ be an irreducible reduced curve. Let

$$
\Pi=\bigcap_{y \in C^{0}} \mathbb{T}_{y} X
$$

where $C^{0} \ni x$ is an open subset of $C$. Assume that:

- $\operatorname{dim} \Pi=k-1$;

- the general tangent hyperplane to $x$ is not tangent to $X$ at other points of $C$.

Then $C$ is a line.

Proof. Let $p: \mathbb{P}^{N} \rightarrow \mathbb{P}^{N-k-2}$ be the projection from $\Pi$. Then $p\left(\mathbb{T}_{y} X\right)$ is a point for any $y \in C^{0}$. Let

$$
\Gamma=\overline{p\left(\bigcup_{y \in C^{0}} \mathbb{T}_{y} X\right)}
$$

By hypothesis the general tangent hyperplane at $x$ is tangent only at $x$. Therefore $\Gamma$ is a line and

$$
\bigcup_{y \in C} \mathbb{T}_{y} X=\mathbb{P}^{k+1}
$$

Let $\Phi: X \rightarrow \mathbb{G}(k, N)$ be the Gauss map. Then by Equation (4) and hypothesis (3), $\Phi(C)$ is a line. The morphism $\Phi$ is an isomorphism in a neighborhood of $x$ and is given by a sublinear system of $\left|K_{X}+(k+1) \mathcal{O}_{X}(1)\right|$, therefore

$$
\left(K_{X}+(k+1) \mathcal{O}_{X}(1)\right) \cdot C=1 .
$$

This proves that $\left(K_{X}+k \mathcal{O}_{X}(1)\right) \cdot C=1-\operatorname{deg} C \leq 0$. Therefore $K_{X}+k \mathcal{O}_{X}(1)$ is not ample. By adjunction theory, [1], the variety $X$ is either a scroll over a curve or an hyperquadric. In both cases $K_{X}+k \mathcal{O}_{X}(1)$ is nef. In particular $\operatorname{deg} C=1$.

Remark 2.11. In the Appendix I derive some further consequences from this relation between adjunction theory and the Gauss map.

Proof of Theorem 2.8. By definition a general fibre is an equivalence class; let $x \in F$ be a general point. By Theorem 2.6 I have the following inclusion $W_{x} \cap F \subset \mathbb{T}_{x} F \cap F$. In particular to prove the thesis it is enough to show that $W_{x} \cap F \neq \emptyset$. Condition i) is now clear. Assume that condition ii) is satisfied. Let $F$ be a general fiber of $\pi^{0}$. Then $F=W_{x}$ and the morphism $p: U \rightarrow X$ is birational. This forces the existence of a curve say $C_{F}$ such that for $y \in C_{F}$ I have $\mathbb{T}_{y} X \supset W_{x}$. If the 3-fold is not defective then I can conclude by Lemma 2.10 that $W_{x}=F$ is a line. Assume that $X$ is defective, then it is a scroll, [3]. The curve $W_{x}$ is not in the fiber of the scroll structure and therefore a general hyperplane tangent at a general point of $W_{x}$ is not tangent to other points of $W_{x}$. I conclude, again by Lemma 2.10 , that $W_{x}$ is a line.

A usual technique in studying projective varieties is the Apollonius or slicing method. That is instead of studying directly $X$ one studies an hyperplane section $X_{1}$, and then tries to rebuild $X$ from $X_{1}$. In the set up of $T_{W} C$ relation one can encounter problems in doing this. 
Example 2.12. Consider $\mathbb{Q}^{3} \subset \mathbb{P}^{4}$. Then $\mathbb{Q}^{3}$ is $T_{W} C$ but its hyperplane section $\mathbb{Q}^{2}$ is only $T C$. Indeed the two rulings belong to disconnected components of the Hilbert scheme of lines on $\mathbb{Q}^{2}$ but they "glue" in $\mathbb{Q}^{3}$.

The really remarkable fact is that this is the only example of "bad" behavior. Let us start with the following observation.

Proposition 2.13 (Hyperplane slicing). Let $X \subset \mathbb{P}^{N}$, and $\mathcal{W}=\left(\mathbb{T}_{x} X \cap X\right)_{\text {red. }}$ Let $X_{1}$ be a general hyperplane section. Then $\mathcal{W}_{1}=\left(\mathbb{T}_{x} X_{1} \cap X_{1}\right)_{\mathrm{red}}$ is an hyperplane section of $\mathcal{W}$.

The next theorem is the result I need to work efficiently with the slicing methods.

Theorem 2.14. Let $X \subset \mathbb{P}^{N}$ be a smooth variety and $X_{1}$ a general hyperplane section. Assume that $X$ is $T_{W} C$ and $\operatorname{dim} W>1$. Then $X_{1}$ is $T_{W_{1}} C$ unless $X \cong \mathbb{Q}^{3} \subset \mathbb{P}^{4}$, where $W_{1}$ is a hyperplane section of $W$.

Remark 2.15. Note that Theorem 2.14 is less appealing if we look for $T C$ instead of $T_{W} C$. The hyperplane section of a $T C$ variety is in general not $T C$. For instance one can consider either a Segre embedding of $\mathbb{P}^{r} \times \mathbb{P}^{1}$ or appropriate subvarieties in $\mathbb{P}^{r} \times \mathbb{P}^{2}$.

To prove Theorem 2.14 I need some results on tangentially connected varieties of low dimension. I prefer to postpone their proofs in the next section.

\section{Tangentially connected varieties of low dimensions}

I keep notations and terminology introduced in Section 1. In particular, to any irreducible component $W \subset\left(\mathbb{T}_{x} X \cap X\right)_{\text {red }}$ it is associated:

- a dominant family of cycles $V$, with universal family $U$ and

$$
V \stackrel{q}{\leftarrow} U \stackrel{p}{\rightarrow} X
$$

- an algebraic relation $T_{W} R(X)$,

- $T_{W} R(X)$-morphism and maps.

Let us start with a simple observation.

Remark 3.1. The only curve with positive tangential restriction is a line. This easily extends in all dimension. That is the only variety contained in its projective tangent space is a linear space.

The first interesting case is that of surfaces. The following is just a modification of a classical result of del Pezzo, [2].

Theorem 3.2. Let $S \subset \mathbb{P}^{N}$ be a surface with positive tangential restriction. Then either $S$ is a scroll or $S \subset \mathbb{P}^{3} \subset \mathbb{P}^{N}$, possibly a plane. In particular the only TC surfaces are surfaces in $\mathbb{P}^{3}$. Furthermore the only TC surface which is not $T_{W} C$ is the quadric. 
Proof. Let $\pi^{0}: S^{0} \rightarrow Z^{0}$ be any $T_{W} R(S)$-morphism. Then either $S^{0}$ is $T_{W} C$ or by Theorem 2.8 and Remark 3.1 the general fibre is a line. The latter immediately implies that $S$ is a scroll, see for instance Theorem 1.4.

We have to classify $T_{W} C$ surfaces. Let $x \in S$ be a general point and $W \subset\left(\mathbb{T}_{x} S \cap\right.$ $S)_{\text {red }}$ an irreducible component. If $W=S$ then $S \cong \mathbb{P}^{2}$. Assume that $\operatorname{dim} W=1$. If $W$ is a line then $W^{2}=1$ and there are infinitely many lines through the general point. This is clearly impossible. To conclude assume that $\langle W\rangle=\mathbb{T}_{x} S$. Since the Gauss map is birational, there are infinitely many curves of the family $V$ passing through $x$, and $\mathbb{S}_{x}=S$, see Definition 1.2. Let us now consider the projection $\varphi: \mathbb{P}^{N} \supset S \rightarrow \mathbb{P}^{N-3}$ from $\mathbb{T}_{x} S$. Let $W_{y} \subset \mathbb{T}_{y} X$ be the general curve in $V$ containing $x$. Then $\mathbb{T}_{x} W_{y} \subset\left(\mathbb{T}_{x} S \cap \mathbb{T}_{y} S\right)$, therefore $\varphi\left(T_{y} S\right)=\varphi\left(W_{y}\right)=\{p t\}$. So that $\operatorname{dim} \varphi(S) \leq 1$.

On the other hand by Theorem 1.5 the general tangent hyperplane at $x$ is smooth elsewhere. Therefore $\operatorname{dim} \varphi(S)=0$ and $S \subset \mathbb{P}^{3}$. I stress that the quadric is the only surface in $\mathbb{P}^{3}$ which is not $T_{W} C$. Indeed it is the only smooth surface in $\mathbb{P}^{3}$ with reducible tangential restriction.

Remark 3.3. Note that in higher dimension there are many $T C$ varieties that are not $T_{W} C$. It is enough to consider either varieties ruled by lines in two different ways or divisors of appropriate Segre embeddings. This is one of the reasons for which I prefer to work with $T_{W} C$ instead of $T C$, see also Remark 2.15.

Theorem 3.2 naturally generalizes to classify varieties with positive dimensional tangential restriction and $\operatorname{cod}_{X} W=1$.

Corollary 3.4. Let $X \subset \mathbb{P}^{N}$ be an n-fold with positive dimensional tangential restriction. Assume that $\operatorname{cod}_{X}\left(\mathbb{T}_{x} X \cap X\right)=1$. Then either $X$ is a scroll over a curve or $\langle X\rangle=\mathbb{P}^{n+1} \subset \mathbb{P}^{N}$.

Proof. By hyperplane slicing we know that a surface section $S$ has positive dimensional tangential restriction, see Proposition 2.13. Therefore by Theorem $3.2 S$ is either a scroll or a surface in $\mathbb{P}^{3}$. The latter is a linear section of an hypersurface. Assume that $S$ is a scroll. Let $W_{x} \subset\left(\mathbb{T}_{x} X \cap X\right)$. Then $W_{x}$ is either a cone with vertex $x$ or a linear space. If $W_{x}$ is a linear space then $X$ is a scroll over a curve by Theorem 1.4. Assume that $W_{x}$ is a cone. Then $S$ is a surface admitting at least two scroll structures. That is $S$ is the quadric surface and $X \cong \mathbb{Q}^{n} \subset \mathbb{P}^{n+1} \subset \mathbb{P}^{N}$.

I am ready to prove Theorem 2.14 .

Proof of Theorem 2.14. Assume first that $\operatorname{dim} X>3$. Let $\pi_{1}^{0}: X_{1}^{0} \rightarrow Z_{1}^{0}$ be the $T_{W_{1}} C(X)$-morphism. If $\operatorname{dim} Z_{1}^{0}>0$ let $D \in \operatorname{Pic}\left(Z_{1}\right)$ be an effective divisor. Let $D_{1}=\overline{\left(\left(\pi_{1}^{0}\right)_{*}^{-1}(D)\right)} \in \operatorname{Pic}\left(X_{1}\right)$ be the strict transform. Then by the Lefschetz Theorem on hyperplane sections, see for instance [9], there is a line bundle $D_{X} \in \operatorname{Pic}(X)$ such that $D_{X \mid X_{1}} \sim D_{1}$. Let $C \subset X_{1} \subset X$ be an hyperplane curve section of $W$. Since $\pi_{1}^{0}$ is proper then $D_{X} \cdot C=D_{1} \cdot C=0$. So that $X$ is not $T_{W} C$. If $\operatorname{dim} X=3$ and $\operatorname{dim} W=2$ then by Corollary $3.4 X$ is either a scroll over a curve or a hypersurface. If $X$ is a scroll and $W$ is a fiber then $X$ is not $T_{W} C$. As already observed the only surface in $\mathbb{P}^{3}$ which is not $T_{W} C$ is the quadric. 
My next aim is to study 3-folds with positive dimensional tangential restriction, or equivalently varieties with $\operatorname{cod}_{X} W=2$.

Let me fix such a 3-fold $X$ with $\operatorname{dim} W=1$. Let $\pi^{0}: X^{0} \rightarrow Z^{0}$ be the $T_{W} R(X)$ morphism and $\pi: X \rightarrow Z$ a $T_{W} R(X)$-map. I distinguish cases according to the dimension of $Z$.

Case 3.5 $(\operatorname{dim} Z=2)$. By Theorem 2.8 and Remark 3.1 the general fibre is a line; note that $x \in W_{x}$. To better understand these varieties let me start with the following result.

Theorem 3.6. Let $X$ be a $k$-fold with positive dimensional tangential restriction. Let

$$
V \stackrel{q}{\leftarrow} U \stackrel{p}{\rightarrow} X
$$

be the incidence variety and $\pi: X \rightarrow Z$ an associated $T_{W} R(X)$ map. Assume that $\operatorname{dim} Z=\operatorname{dim} X-1$ and $x \in W_{x}$. Then $p$ is birational. Let $d=\operatorname{dim} p(\operatorname{Exc}(p))$, if $\operatorname{Exc}(p) \neq \emptyset$ then $d \geq k / 2$. If equality is fulfilled then $\pi$ is a morphism, the contraction of an extremal ray, and the irreducible components of each fibre are projective spaces.

Remark 3.7. It is not true in general that $\pi$ extends to a scroll structure on $X$. Consider

$$
X=\left(x_{0} y_{0}+x_{1} y_{1}+x_{2} y_{2}=0\right) \subset \mathbb{P}^{2} \times \mathbb{P}^{3} \subset \mathbb{P}^{11} .
$$

Let $W$ be a line. Then the $T_{W} R(X)$ fibration is the projection on $\mathbb{P}^{3}$ and has an isolated fibre $F_{0} \cong \mathbb{P}^{2}$.

Proof. Let $V^{n}$ and $U^{n}$ be the normalizations of $V$ and $U$ respectively. By abuse of notation let

$$
V^{n} \stackrel{q}{\leftarrow} U^{n} \stackrel{p}{\rightarrow} X,
$$

the induced morphisms. By Theorem 2.8 and Remark 3.1 the general fibre $F$ of $\pi$ is a line. So that $p$ is birational and $q$ is a scroll structure on $V^{n}$, [7, II.2.8]. Assume that $p$ is not an isomorphism. The variety $X$ is smooth, therefore $\operatorname{Exc}(p)$ has pure codimension 1, [7, VI.1.5]. Let $D \subset U^{n}$ be the $p$-exceptional Cartier divisor. By construction $p$ is an isomorphism on the general fiber of $\pi$. Therefore $D$ does not intersect the general fibre of $q$. And $D=q^{*} M$ for some Cartier divisor $M \in \operatorname{Pic}\left(V^{n}\right)$. So that $p(D)$ is covered by a $(\operatorname{dim} X-2)$-dimensional family of lines. Then by Theorem 1.4

$$
\operatorname{dim} X-2 \leq 2 d-2 .
$$

Furthermore if equality is fulfilled then $p(\operatorname{Exc}(p))$ is pure dimensional and each irreducible component of $p(D)$ is a projective space. Assume that $d=\operatorname{dim} X / 2$. Then $p(D)$ is covered by finitely many $\mathbb{P}^{d}$. Let $\mathcal{A}$ be a very ample linear system on $V$ and $\mathcal{A}_{X}=p_{*} q^{*} \mathcal{A}$. Then

$$
\operatorname{Bsl} \mathcal{A}_{X} \subseteq p(D)=\cup \mathbb{P}^{d} .
$$

Let $A_{X} \in \mathcal{A}_{X}$ be a general element and $F$ a general fiber of $\pi$. Then by construction

$$
A_{X} \cdot F=0 .
$$


Let $Z \subset X$ be any curve. If $Z$ is not proportional to $F$, then $Z \not \subset p(D)$, and by Equation (6)

$$
A_{X} \cdot Z>0 \text {. }
$$

This means that $[F]$ is on the boundary of the cone of effective curves. Furthermore by looking at the birational morphism $p$ we see that

$$
K_{X} \cdot F=-2 .
$$

So that $[F]$ is an extremal ray and the contraction of $[F]$, see [5], gives the desired morphism.

An immediate consequence of Theorem 3.6 is the following.

Corollary 3.8. If $X$ is a 3-fold and $Z$ a surface, then $\pi: X \rightarrow Z$ is a scroll structure onto a smooth surface.

The above corollary yields.

Corollary 3.9. Assume that $X \subset \mathbb{P}^{N}$ is an $n$-fold with positive dimensional tangential restriction. Assume that $\operatorname{cod}_{X}\left(\mathbb{T}_{x} X \cap X\right)=2$ and $\operatorname{dim} Z=2$. Then $\pi^{0}: X^{0} \rightarrow Z^{0}$ extends to a scroll structure onto a smooth surface.

Proof. Let $T$ be a smooth 3-fold hyperplane section of $X$. By Corollary $3.8 \pi_{\mid T}^{0}$ extends to a scroll structure $\pi_{T}: T \rightarrow Z$ onto a smooth surface $Z$. Then the general fibre $F$ of $\pi^{0}: X^{0}-\rightarrow Z^{0}$ is a $\mathbb{P}^{n-2}$, and $K_{X \mid F} \sim \mathcal{O}(n-1)$. By adjunction theory, [1], $\pi: X \rightarrow Z$ is a scroll structure over a smooth surface.

Case 3.10 $(\operatorname{dim} Z=1)$. In this case I cannot apply Theorem 2.8. For this reason I am forced to assume that $x \in W_{x}$.

Proposition 3.11. If $\operatorname{dim} X=3$, with $x \in W_{x}$ and $\operatorname{dim} Z=1$, then $\pi^{0}$ extends to a morphism, and $X$ is a divisor in a, possibly singular, scroll over a smooth curve.

Proof. One can always assume that $Z$ is smooth. Let $M \in \operatorname{Pic}\left(Z^{0}\right)$ be a spanned line bundle. Then $\left(\pi^{0}\right)^{*} M \in \operatorname{Pic}(X)$ defines a morphism $\pi: X \rightarrow Z$. Let $F \subset X$ be a general fiber then by hypothesis and Theorem $2.8 F$ is $T C$. Therefore by Theorem $3.2 F$ is a surface in $\mathbb{P}^{3}$. Then $P=\bigcup_{z \in Z}\langle F\rangle$ is a 4-fold scroll, see Theorem 1.4, and $X \in P$ is a divisor.

The higher dimensional case is immediate.

Corollary 3.12. Assume that $X \subset \mathbb{P}^{N}$ is an $n$-fold with positive tangential restriction. Assume that $\operatorname{cod}_{X}\left(\mathbb{T}_{x} X \cap X\right)=2, x \in W_{x}$ and $\operatorname{dim} Z=1$. Then $\pi$ extends to $a$ morphism onto a smooth curve, and $X$ is a divisor in a possibly singular scroll onto a smooth curve. 
Case 3.13 $(\operatorname{dim} Z=\mathbf{0})$. In this case $X$ is a $T_{W} C$ 3-fold. Together with low codimension varieties essentially new varieties appear. That is $T_{W} C$ varieties covered by lines. The main distinction can be traced in the morphism $p: U \rightarrow X$. I study the case in which $p$ is generically finite.

Theorem 3.14. Let $X$ be a $T_{W} C$ 3-fold and

$$
V \stackrel{q}{\leftarrow} U \stackrel{p}{\rightarrow} X
$$

the incidence variety. Assume that $p$ is generically finite. Then $W_{x}$ is a line, $\operatorname{rk} \operatorname{Pic}(X)=$ 1 and $X$ is a del Pezzo 3-fold of degree 4 or 5 embedded by $|\mathcal{O}(1)|$.

Proof. The morphism $p$ is generically finite. Then $W_{x}$ is contained in infinitely many tangent spaces and by Lemma $2.10 W_{x}$ is a line.

Then I prove that $\operatorname{rk} \operatorname{Pic}(X)=1$. This is a standard argument, [7, IV. 3.13.3], but I sketch it here for the convenience of the reader. As in the proof of Theorem 3.6, let $U^{n}$ and $V^{n}$ the normalizations of $U$ and $V$, with induced maps

$$
V^{n} \stackrel{q}{\leftarrow} U^{n} \stackrel{p}{\rightarrow} X .
$$

Then $q$ is a scroll structure. The idea is to prove that any divisor $G$ such that $G \cdot W_{x}=0$ is numerically equivalent to zero. The way is to go up and down using the morphisms $p$ and $q$ extending the numerical vanishing to $S_{W_{x}}, S_{S_{W_{x}}}$ and so on. This sequence covers all of $X$ after finitely many steps because $X$ is $T_{W} C$.

Since $\operatorname{Pic}(X)=\mathbb{Z}$ and $X$ is covered by lines, $X$ is Fano. The morphism $p$ is dominant therefore the general normal bundle of $W_{x}$ is spanned and

$$
K_{X} \cdot W_{x} \leq-2 .
$$

Then the Fano index of $X$ is 2 and $X$ is a del Pezzo 3-fold. Looking at Fujita's list, [4], the only ones that satisfy our requirement are those of degree 4 and 5 .

Remark 3.15. It is important to stress a difference between the two varieties appearing in Theorem 3.14. The degree 4 del Pezzo is a codimension 2 threefold. Then it has to have a positive dimensional tangential restriction. The degree 5 is embedded in $\mathbb{P}^{6}$. The positive dimensional tangential restriction is only due to the existence of lines on $X$. This 3 -fold is a linear section of a homogeneous manifold.

When $p: U \rightarrow X$ is not generically finite the situation is more intricate and I am not able to complete the classification. Let us observe the following.

Proposition 3.16. Let $X \subset \mathbb{P}^{n}$ be a smooth 3-fold and $x \in X$ a general point, and assume that:

(i) there are no lines through $x$;

(ii) there is a surface $S_{x} \ni x$ such that $\left\langle S_{x}\right\rangle=\mathbb{P}^{3}$;

(iii) $S_{x} \cap S_{y} \neq \emptyset$;

(iv) $S_{x} \neq S_{y}$ for $x \neq y$.

Then $X \subset \mathbb{P}^{4}$. 
Proof. By Noetherianity I can assume that all such surfaces are linearly equivalent. Let $S_{1} \cap S_{2}=C$ be a general complete intersection and $S_{\lambda}$ an element of the pencil $\left|S_{1}, S_{2}\right|$.

Assume that $X \neq \mathbb{P}^{3}$. Then by hypothesis $C$ is not a line and it is planar. Moreover I have

$$
\bigcap_{\lambda}\left\langle S_{\lambda}\right\rangle=\langle C\rangle=\mathbb{P}^{2} .
$$

Assume first that $\mathbb{T}_{x} X \supset\langle C\rangle$. Then $T_{x} S_{\lambda}=\langle C\rangle$ and all $S_{\lambda}$ are not transverse to each other at each intersection point. To conclude observe that if $\operatorname{dim} \mathbb{T}_{x} X \cap\langle C\rangle=1$ then $X \subset\left\langle\mathbb{T}_{x} X,\langle C\rangle\right\rangle=\mathbb{P}^{4}$.

Remark 3.17. Note that the assumption on lines is crucial. It is enough to consider a linear system of quadrics in the Segre embedding of $\mathbb{P}^{1} \times \mathbb{P}^{2}$.

This proposition suggests that $T C$ 3-folds with not generically finite $p: U \rightarrow X$ are not covered by varieties of small codimension.

Conjecture 3.18. Let $X \subset \mathbb{P}^{n}$ be a smooth TC 3-fold and assume that $p: U \rightarrow X$ is not generically finite. Then $W$ is not planar, and $\langle X\rangle=\mathbb{P}^{5}$.

Remark 3.19. The conjecture seems quite hard. Note that if $X$ is not of codimension 2 a general hyperplane section is a surface with excess trisecant lines. The classification of the latter is another difficult open problem in classical projective geometry. I do not expect that such surfaces can be hyperplane sections of a smooth $T C 3$-fold. Note further that smoothness is crucial. If one drops it divisors in scrolls appears.

\section{A Projective interpretation of modern adjunction theory}

Classic adjunction theory is a way to study embedded projective varieties, through their hyperplane sections. In recent times the accent of this theory moved towards pairs of variety and ample line (vector) bundle. For a complete account of both aspects and also for the precise definition of the varieties involved in the following theorems see [1].

Here I work in the opposite direction. That is recover some projective information using generalised adjunction theory.

Let $X \subset \mathbb{P}^{n}$ be a nonlinear smooth $k$-fold, and $G(X) \subset \mathbb{P}^{N}$ its image under the Gauss map. It is well known that, [10], the morphism $G: X \rightarrow G(X)$ is birational and is given by a sublinear system of $\left|K_{X}+(k+1) L\right|$, where $L=\mathcal{O}_{X}(1)$. This proves that $K_{X}+$ $(k+1) L$ is ample unless $(X, L) \cong\left(\mathbb{P}^{k}, \mathcal{O}(1)\right)$. The latter is a well known adjunction theoretic statement. The proof of Lemma 2.10 is based on this simple observation. Here I want to go a bit further.

It is strange but, to the best of my knowledge, the following characterisation of smooth scrolls over a curve has not been noticed yet.

Theorem A.1. Let $X \subset \mathbb{P}^{n}$ be a smooth $k$-fold. Let $G(X) \subset \mathbb{P}^{N}$ be the image of the Gauss map of $X$, embedded via the Plücker embedding. Then $G(X)$ contains a line not 
contained in $\operatorname{Sing}(G(X))$ if and only if $X$ is either a scroll over a curve or an hyperquadric.

Proof. One direction is immediate. In both cases $X$ is covered by curves $C$ on which $\left(K_{X}+(k+1) L\right) \cdot C=1$. Assume that $G(X)$ contains such a line. The Gauss map can be viewed as the normalization of $G(X)$. The line not contained in $\operatorname{Sing}(G(X)$ is then birationally covered by a curve $C \subset X$ such that $\left(K_{X}+(k+1) L\right) \cdot C=1$. In particular $\left(K_{X}+k L\right) \cdot C=1-L \cdot C \leq 0$ is not ample. This shows by adjunction theory, see for instance [1, p. 151], that $X$ is either a scroll or an hyperquadric.

Remark A.2. The assumption that $l \not \subset \operatorname{Sing}(G(X))$ is crucial. Think of any surface in $\mathbb{P}^{3}$ containing a line. It would be natural to expect that the above statement is true for normal $k$-fold with a nondegenerate Gauss map. The theoretic adjunction proof works only under the Gorenstein assumption on $X$. Note that this also gives strong geometric restrictions for varieties that are the image via the Gauss map of a smooth variety. For instance any variety of dimension $k$ containing a family of lines, not contained in $\operatorname{Sing}(Y)$, of dimension $h$ is not the image of the Gauss map of a smooth variety if $h \neq 2 k-3$.

To simplify the notation I introduce the following invariants of $(X, L)$.

Definition A.3. Let $X \subset \mathbb{P}^{n}$ be a $k$-fold and $G(X)$ the image of the Gauss map. Let

$$
\delta(X)=\min \{d \in \mathbb{N} \mid \text { there exists a curve } D \not \subset \operatorname{Sing}(G(X)) \text { of degree } d\}
$$

and

$$
L \delta(X)=\overline{\left(\bigcup_{\operatorname{deg} Z=\delta(X)} Z\right)} \subset G(X) .
$$

One can similarly obtain the following theorem, cf. [1, Theorem 7.5.2, Theorem 7.5.3].

Theorem A.4. Let $X \subset \mathbb{P}^{n}$ be a smooth $k$-fold. Let $G(X) \subset \mathbb{P}^{N}$ be the image of the Gauss map of $X$, embedded via the Plïcker embedding.

If $\delta(X)=2$ and $L \delta(X)=G(X)$ then $X$ is one of the following:

- del Pezzo variety;

- quadric fibration over a curve;

- scroll over a surface.

If $\delta(X)=2$ and $L \delta(X) \neq G(X)$ then $X$ is the blow up in a point of a smooth variety and $L \delta(X)=\nu_{2}\left(\mathbb{P}^{k-1}\right)$.

If $\delta(X)=3$ and $L \delta(X)=G(X)$ then $X$ is one of the following:

- Mukai variety;

- del Pezzo fibration over a curve;

- quadric fibration over a surface;

- scroll over a 3-fold;

- the Veronese surface.

For any variety in the lists $\delta(X)$ is the one expected. 
Proof. Let $D \subset G(X)$ be a curve with $\operatorname{deg} D=\delta(X)$ and $D \not \subset \operatorname{Sing}(X)$. Then there exists a curve $C \subset X$ with $\left(K_{X}+(k+1) L\right) \cdot C=\delta(X)$. If $\delta(X)=2$ then

$$
\left(K_{X}+k L\right) \cdot C=2-\operatorname{deg} C
$$

and I claim that $\operatorname{deg} C=1$. If $\operatorname{deg} C \geq 2$ then $K_{X}+k L$ is not ample and $X$ has to be either a scroll or an hyperquadric. This contradicts the minimality of $\delta(X)$ and proves that $C$ is a line. Therefore $K_{X}+k L$ is ample and $K_{X}+(k-1) L$ is not ample. Then by [1, Theorem 7.2.4] and [1, Theorem 7.5.2] I conclude.

If $\delta(X)=3$ and $L \delta(X)=G(X)$ then through the general point of $X$ there exists a curve $C \subset X$ and this time $\operatorname{deg} C \leq 2$. If $\operatorname{deg} C=1$ I conclude as above by [1, Theorem 7.5.3]. If $\operatorname{deg} C=2$ then $\left(K_{X}+k L\right) \cdot C=1$ and $\left(K_{X}+(k-1) L\right) \cdot C=-1$. Therefore $X$ is the Veronese surface by [1, Theorem 7.2.4].

\section{References}

[1] M. C. Beltrametti, A. J. Sommese, The adjunction theory of complex projective varieties. De Gruyter 1995. MR1318687 (96f:14004) Zbl 0845.14003

[2] P. del Pezzo, Sugli spazi tangenti ad una superficie o ad una varietà immersa in uno spazio a più dimensioni. Rend. Acc. Napoli, 25 (1886), 176-180. JFM 18.0450.01

[3] L. Ein, Varieties with small dual varieties. I. Invent. Math. 86 (1986), 63-74. MR853445 (87m:14047) Zbl 0603.14025

[4] T. Fujita, Classification theories of polarized varieties. Cambridge Univ. Press 1990. MR1162108 (93e:14009) Zbl 0743.14004

[5] Y. Kawamata, K. Matsuda, K. Matsuki, Introduction to the minimal model problem. In: Algebraic geometry, Sendai, 1985, volume 10 of Adv. Stud. Pure Math., 283-360, North-Holland 1987. MR946243 (89e:14015) Zbl 0672.14006

[6] S. L. Kleiman, About the conormal scheme. In: Complete intersections (Acireale, 1983), volume 1092 of Lecture Notes in Math., 161-197, Springer 1984. MR775882 (87g:14060) Zbl 0547.14031

[7] J. Kollár, Rational curves on algebraic varieties. Springer 1996. MR1440180 (98c:14001) Zbl 0877.14012

[8] B. Segre, Sulle $V_{n}$ contenenti più di $\infty^{n-k} S_{k}$. I. Atti Accad. Naz. Lincei. Rend. Cl. Sci. Fis. Mat. Nat. (8) 5 (1948), 193-197. MR0036041 (12,49f) Zbl 0032.30803

[9] A. J. Sommese, On manifolds that cannot be ample divisors. Math. Ann. 221 (1976), 55-72. MR0404703 (53 \#8503) Zbl 0306.14006

[10] F. L. Zak, Tangents and secants of algebraic varieties, volume 127 of Translations of Mathematical Monographs. Amer. Math. Soc. 1993. MR1234494 (94i:14053) Zbl 0795.14018

Received 11 September, 2007; revised 18 December, 2007

M. Mella, Dipartimento di Matematica, Università di Ferrara, Via Machiavelli 35, 44100 Ferrara, Italia

Email: mll@unife.it 